\title{
Trough Peak Ratio
}

National Cancer Institute

\section{Source}

National Cancer Institute. Trough Peak Ratio. NCI Thesaurus. Code C122339.

The concentration at the start of a dosing interval divided by the maximum concentration during the dosing interval. 